\title{
Optimizing of Raman Gain and Bandwidth for Dual Pump Fiber Optical Parametric Amplifiers Based on Four-Wave Mixing
}

\section{El-khashab HK ${ }^{1}$, Mustafa FM $^{2 *}$ and Barakat TM ${ }^{1}$}

${ }^{1}$ Electrical Engineering Department, Faculty of Engineering, Fayoum University, Fayoum, Egypt

${ }^{2}$ Electrical Engineering Department, Faculty of Engineering, Beni-Suef University, Beni-Suef, Egypt

\begin{abstract}
Fiber optical parametric amplifiers (FOPAs) are very important for future fiber optical amplifiers because of their high gains, broad gain bandwidth and relatively low noise figure. Recently, Fiber-optic parametric amplifiers (FOPAs), which are based on Four-Wave Mixing (FWM) occurring inside optical fiber, have got lots of attentions due to its wide gain bandwidth, flat gain spectrum and low noise because they can provide broadband amplification and can thus replace erbium-doped fiber amplifier used commonly for signal amplification. In this paper, we proposed an efficient dual pump optical parametric amplifiers which enjoy the following new features: (1) providing a uniform gain over a relatively wide bandwidth when they are pumped at two wavelengths located on each side of Zero Dispersion WaveLength (ZDWL), (2) Maximizing repeater spacing and (3) providing broadband and high gain in high speed long-haul wavelength division multiplexing (WDM) transmission. Our results show that the maximum gain is $61.6454 \mathrm{~dB}$ and broadband is $350 \mathrm{~nm}$ compared with previous works which the gain is computed over the spectral optical wavelengths $(1.35 \mu \mathrm{m} \leq \lambda$ signal $\leq 1.75 \mu \mathrm{m})$.
\end{abstract}

Keywords: Fiber optical parametric amplifiers (FOPAs); Wavelength division multiplexing (WDM); Dual pump FOPA; Four-wave mixing (FWM)

\section{Introduction}

The optical amplifier played a crucial role in the communications revolution that began two decades ago. The development of optical fiber optic amplifiers (FOBA) has significantly increased the transmission capacity of fiber communication systems [1]. Fiber optic optical amplifiers (FOAs) with flat gain spectra and wide bandwidth are very promising for fully optical signal processing applications such as signal generation, broadband conversion, optical sampling, switching, and wavelength division multiplexing (WDM) [2].

The optical amplifiers are of great importance to fiber optic amplifiers in the future due to their high gain, wide bandwidth and relatively low noise value. FOAs can be used as an optical amplifier as well as in signal processing such as waveform conversion, optical multiplexing, sampling, and reduction. Fiber-optic amplifiers (FOAs), which are based on mixing four waves within optical fibers, attract considerable attention as they can provide amplification of the broadband, and thus can replace the erbium fiber amplifier commonly used to amplify the signal [3]. The most important feature of the FOA double pump is that it can provide relatively flat gains on a much wider bandwidth than is possible with a single FOA pump [4]. Recently, the optical fiber parametric amplifier (FOPA) which relies on four-wave nonlinear processing mixing has received a lot of attention because of its wide gain bandwidth, flat spectrum gain and low noise [5]. In the present paper, we processed: gain and bandwidth of dual pump optical parametric amplifier and also, we show a parameters affecting on dual pump optical parametric amplifier gain and bandwidth to obtain a large and broadened bandwidth. The gain is computed over the spectral optical wavelengths $(1.35 \mu \mathrm{m} \leq \lambda$ signal $\leq 1.75 \mu \mathrm{m})$.

\section{Proposed Model of Dual-Pump FOPA}

Fiber Optical parametric amplifiers are based on Four Wave Mixing (FWM) effect which transfers power from strong pump fields to weak signal and idler fields. Governed by conservation of energy principle idler generation is expressed as $[1,3,6]$ : $\omega \mathrm{i}=\omega \mathrm{a}+\omega \mathrm{b}-\omega \mathrm{s}$

Where, $\omega_{a}, \omega_{b}, \omega_{s}$ and $\omega_{i}$ are two pump frequencies, signal frequency and the idler frequency.

The parametric signal gain $(G)$ in dual-pump FOPA configuration is given by eqns. (2) and (3).

$$
G\left(\omega_{3}\right)=\left[1+\left(1+K^{2} / 4 g^{2}\right) \sinh ^{2}(g l)\right] \times e^{-\alpha l},
$$

where $g$ is the gain coefficient shown in eqn. (2), $\gamma$ is the nonlinear coefficient and $L$ is the fiber length [7].

$$
g^{2}=4 \gamma^{2} P_{1} P_{2}-\left(\frac{K}{2}\right)^{2}
$$

The parametric amplification is governed by phase matching condition given as:

$$
K=\Delta \beta+\gamma\left(P_{1}+P_{2}\right)
$$

Where ' $\gamma$ ' is non-linear co-efficient of the fiber and $P_{1}$ and $P_{2}$ are powers of the pumps used, $\Delta \beta$ is linear phase mismatch while $2^{\text {nd }}$ term represents non-linear phase mismatch. For perfect phase mismatch, total phase ' $\mathrm{K}=0$ ' which gives maximum gain and is achievable around ZDWL. The power growth in both signal and idler is assumed to be same by Manley-Rowe relation, leading to equal power depletion in both the pumps [8].

$$
\Delta \beta=\beta_{3}\left(\omega_{c}-\omega_{0}\right)\left[\left(\omega_{s}-\omega_{c}\right)^{2}-\omega_{d}^{2}\right]
$$

${ }^{*}$ Corresponding author: Mustafa FM, Electrical Engineering Department, Faculty of Engineering, Fayoum University, Fayoum, Egypt, E-mail: Fmmg80@eng.bsu.edu.eg Received May 15, 2018; Accepted June 01, 2018; Published June 08, 2018

Citation: El-khashab HK, Mustafa FM, Barakat TM (2018) Optimizing of Raman Gain and Bandwidth for Dual Pump Fiber Optical Parametric Amplifiers Based on Four-Wave Mixing. J Telecommun Syst Manage 7: 161. doi: 10.4172/21670919.1000161

Copyright: ( 2018 El-khashab HK, et al. This is an open-access article distributed under the terms of the Creative Commons Attribution License, which permits unrestricted use, distribution, and reproduction in any medium, provided the original author and source are credited. 
Where $\omega_{c}=\frac{\omega_{p 1}+\omega_{p 2}}{2}$ and $\omega_{d}=\frac{\omega_{p 1}-\omega_{p 2}}{2}$

As shown in Figure 1, $\omega_{\mathrm{s}}$ and $\omega_{\mathrm{i}}$ are the signal and idler frequencies, respectively. They locate at the positions that the condition of $\omega_{\mathrm{a}}+\omega_{\mathrm{b}}=\omega_{\mathrm{s}}+\omega_{\mathrm{i}}$ is satisfied. The signal and idler gain spectra are symmetric with respect to the center frequency. It is convenient to use $\mathrm{w}_{\mathrm{c}}$ and $\Delta \mathrm{w}_{\mathrm{d}}$ as the two independent parameters, instead of $\mathrm{w}_{1}$ and $\mathrm{w}_{2}$, and to get maximum parametric gain in Equation., the total phase mismatch $\mathrm{K}$ should be equal to zero or when, $\Delta \beta=-\gamma(P 1+P 2)$, and this occurs at signal frequencies that satisfy the well-known phase matching condition [9-11]:

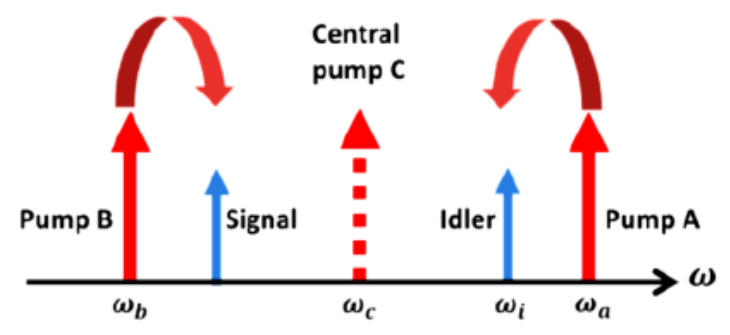

Figure 1: Schematic diagram of dual-pump FOPA configuration.

$\Delta \beta=-\gamma\left(p_{1}+p_{2}\right)=0$,

and the linear phase mismatch $\Delta \beta$ is given by:

$$
\begin{aligned}
& \Delta \beta=\beta_{s}+\beta_{i}-\beta_{p 1}-\beta_{p 2}=\beta_{2}\left[\left(\Delta \omega_{s}\right)^{2}-\left(\Delta \omega_{d}\right)^{2}\right] \\
& \beta_{2} \cong \beta_{3}\left(\omega_{c}-\omega_{o}\right)^{2}
\end{aligned}
$$

Where $\beta_{s}, i, p_{1}, p_{2}$ the signal, idler are, pump one and pump two propagation constants, respectively,

$$
\begin{aligned}
& \mathrm{w}_{\mathrm{c}}=\left(\omega_{1}-\omega_{2}\right) / 2 \\
& \left.\Delta \omega_{\mathrm{s}}=\omega_{3}-\omega_{\mathrm{c}}, \Delta \omega_{\mathrm{d}}=\left(\omega_{1}-\omega_{2}\right) / 2=\omega_{\mathrm{c}}-\omega_{1}=\omega_{2}-\omega_{\mathrm{c}}\right)
\end{aligned}
$$

The linear phase mismatch $\Delta \beta$ in eqn. (5) is expressed by:

$$
\begin{aligned}
& \Delta \beta\left(\lambda_{c}\right)=\left(R_{1}\left(\frac{\Delta \lambda_{c}}{\lambda_{o} \lambda_{c}}\right)\right)\left(\left(\frac{\lambda_{c}-\lambda_{3}}{\lambda_{3} \lambda_{c}}\right)^{2}\right)-\left(\frac{B / 2}{\lambda^{2}{ }_{c}-(\mathrm{B} / 2)^{2}}\right)^{2} \\
& R_{1}=\beta_{3}(2 \pi c)^{3}, \Delta \lambda_{c}=\lambda_{c}-\lambda_{o}, \lambda_{1}=\lambda_{o}-B / 2, \lambda_{2}=\lambda_{o}+B / 2 \text { and } B=\lambda_{2}-\lambda_{1}
\end{aligned}
$$

Where $R_{1}=\beta_{3}(2 \pi c)^{3}, \Delta \lambda_{c}=\lambda_{c}-\lambda_{o}, \lambda_{1}=\lambda_{o}-B / 2, \lambda_{2}=\lambda_{o}+B / 2$ and $B=\lambda_{2}-\lambda_{1}$ is the bandwidth, and $\beta_{3}$ is the third-order dispersion, generally provided by manufacturers, $w_{0}$ is the zero-dispersion wavelength ZDWL. Therefore, adjusting separately each the pump central wavelength, ZDWL and two pump wavelengths, the magnitude and shape of the gain spectrum can be optimized. The $B$ term contributes only when two pumps are used and is independent of the signal and idler frequencies.

\section{Simulation Results and Discussion}

In this section we discuss different parameters that effect on dual pump optical parametric amplifier gain and bandwidth such that pump wavelengths, pump power, non-linear coefficient, phase mismatch, fiber length and attenuation, to obtained maximum gain and bandwidth.

\section{Effect of dual pumping wavelength on gain and bandwidth}

Figure 2, simulated at different values of dual pump wavelengths and the figure draw at assumed set of operating parameters attenuation constant $\alpha=0.1 \mathrm{~dB} / / \mathrm{km}$, pumping power $\mathrm{p}_{1}=0.6 \mathrm{mw}$ and $\mathrm{p}_{2}=0.4 \mathrm{~mW}$, fiber length $L=0.15 \mathrm{~km}$, non-linear coefficient $\gamma=25 \mathrm{w} / \mathrm{km}$ and phase mismatched $\beta=0.0006 \mathrm{~s}^{3} / \mathrm{km}$. In this case we adjustment the dual pumping wavelength with the assumed set of operating parameters to obtain the maximum gain and bandwidth.

From Figure 2, we get the optimum results occurs at dual pump wavelength $\lambda=1546 \mathrm{~nm}$ and $\lambda=1557 \mathrm{~nm}$ where, the maximum gain is $43.9941 \mathrm{~dB}$ and the maximum bandwidth is $290 \mathrm{~nm}$.

\section{Effects of non-linear coefficient on gain and bandwidth}

Figure 3; shown the relation between gain of amplifier and wavelength at different values of non-linear coefficient.

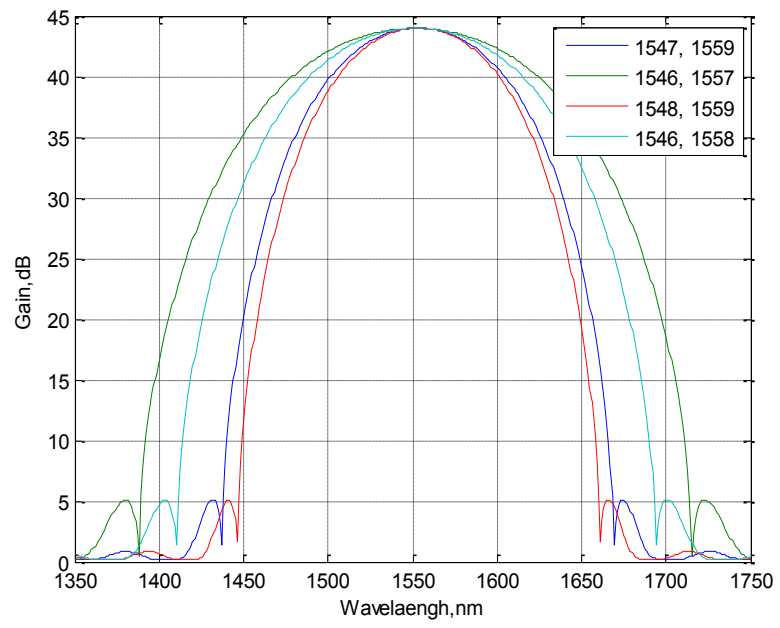

Figure 2: Variations of Gain against wavelength at different values of dua pumping.

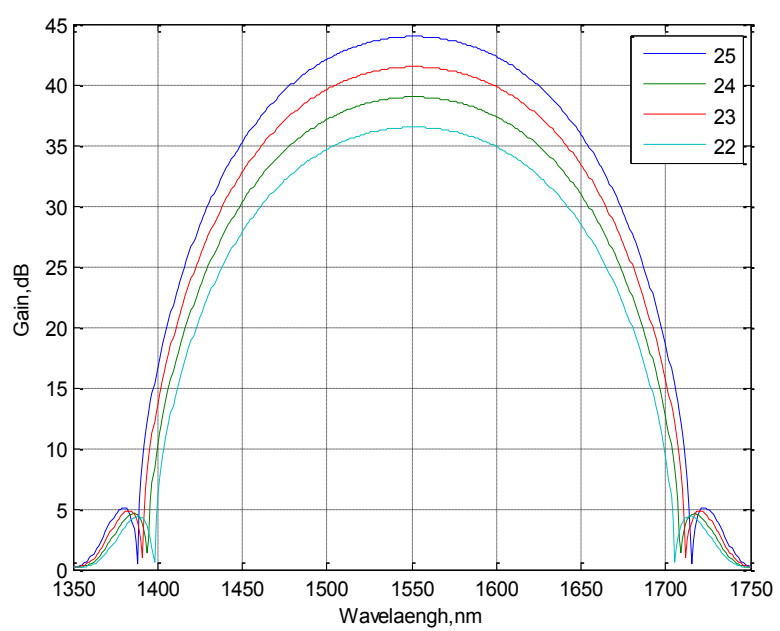

Figure 3: Variations of Gain against wavelength at different values of nonlinear coefficient.

Also from Figure 3, we get the non-linear coefficient $\gamma$ effects on the amplifier gain characteristics. As $\gamma$ increased the maximum gain and bandwidth increased. The figure draw at assumed set of operating parameters attenuation constant $\alpha=0.1 \mathrm{~dB} / \mathrm{km}$, pumping power $\mathrm{p}_{1}=0.6$ mw and $\mathrm{p}_{2}=0.4 \mathrm{~mW}$, fiber length $\mathrm{L}=0.15 \mathrm{~km}$, phase mismatched $\beta=0.0006 \mathrm{~s}^{3} / \mathrm{km}$ and dual pump wavelengths $\lambda_{1}=1546 \mathrm{~nm}$ and $\lambda_{2}=1557$ $\mathrm{nm}$. Also we get maximum gain is $43.966 \mathrm{~dB}$ is attained at the highest value of $\gamma=25 \mathrm{w} / \mathrm{km}$ and the bandwidth is $290 \mathrm{~nm}$. 


\section{Effect of phase mismatch on gain and bandwidth}

Figure 4; shown the relation between gain of amplifier and wavelength at different values of phase mismatch $\beta$.

From Figure 4, we get the coefficient of phase mismatch $\beta$ effects on the amplifier gain characteristics. Approximate the gain constant with beta but the bandwidth has little variation whether increase or decrease of $\beta$. The figure draw at assumed set of operating parameters attenuation constant $\alpha=0.1 \mathrm{~dB} / \mathrm{km}$, pumping power $\mathrm{p}_{1}=0.6 \mathrm{mw}$ and $\mathrm{p}_{2}=0.4 \mathrm{~mW}$, fiber length $\mathrm{L}=0.15 \mathrm{~km}$, non-linear coefficient $\gamma=25 \mathrm{w} / \mathrm{km}$ and dual pump wavelengths $\lambda_{1}=1546 \mathrm{~nm}$ and $\lambda_{2}=1557 \mathrm{~nm}$. In this case the best result has get maximum gain of $44.0293 \mathrm{~dB}$ is attained at $\beta$ equal to $0.0005 \mathrm{~s}^{3} / \mathrm{km}$ and the bandwidth is $319 \mathrm{~nm}$ at the assumed set of operating parameters.

\section{Effect of fiber length on gain and bandwidth}

Figure 5 shows the relation between the amplifier gain and wavelength at different values of fiber length.

The Figure 5, draw at assumed set of operating parameters attenuation constant $\alpha=0.1 \mathrm{~dB} / \mathrm{km}$, pumping power $\mathrm{p}_{1}=0.6 \mathrm{mw}$ and

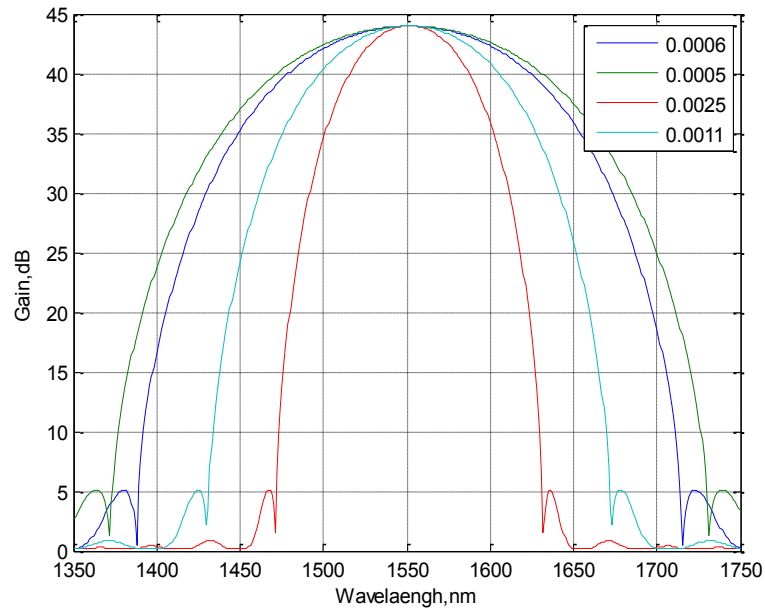

Figure 4: Variations of Gain against wavelength at different values of phase mismatch $\beta$.

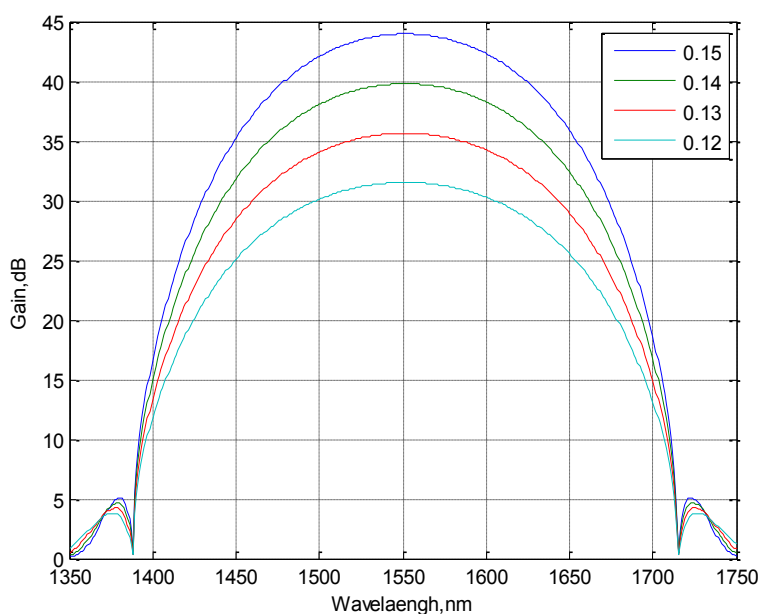

Figure 5: Variations of Gain against wavelength at different values of fiber lengths. $\mathrm{p}_{2}=0.4 \mathrm{~mW}$, dual pump wavelengths $\lambda_{1}=1546 \mathrm{~nm}$ and $\lambda_{2}=1557 \mathrm{~nm}$ non-linear coefficient $\gamma=25 \mathrm{w} / \mathrm{km}$ and phase mismatched $\beta=0.0006 \mathrm{~s}^{3} /$ $\mathrm{km}$. We get Fiber length L effects on the amplifier gain characteristics. As the fiber length increased the maximum gain increased but the bandwidth has a little variation whether increasing or decreasing the fiber length. Maximum gain of $43.966 \mathrm{~dB}$ is attained at fiber length equal to $0.15 \mathrm{~km}$ and the bandwidth is $292 \mathrm{~nm}$. The optimum results occurs at length of fiber $\mathrm{L}=0.15 \mathrm{~km}$.

\section{Effect of attenuation on gain and bandwidth}

Figure 6 shows relation between the amplifier gain and wavelength at different values of the attenuation $\alpha$, where attenuation effects on the amplifier gain characteristics. As the attenuation increased or decreased the maximum gain increased. The bandwidth has a little variation whether increasing or decreasing the attenuation

Figure 6 draw at assumed set of operating parameters fiber length $\mathrm{L}=0.15 \mathrm{~km}$, pumping power $\mathrm{p}_{1}=0.6 \mathrm{mw}$ and $\mathrm{p}_{2}=0.4 \mathrm{~mW}$, dual pump wavelengths $\lambda_{1}=1546 \mathrm{~nm}$ and $\lambda_{2}=1557 \mathrm{~nm}$, non-linear coefficient $\gamma=25$ $\mathrm{w} / \mathrm{km}$ and phase mismatched $\beta=0.0006 \mathrm{~s}^{3} / \mathrm{km}$. The best result has get at $\alpha=0.8 \mathrm{~dB} / \mathrm{km}$ where maximum gain is $44.016 \mathrm{~dB}$ and bandwidth is $296 \mathrm{~nm}$.

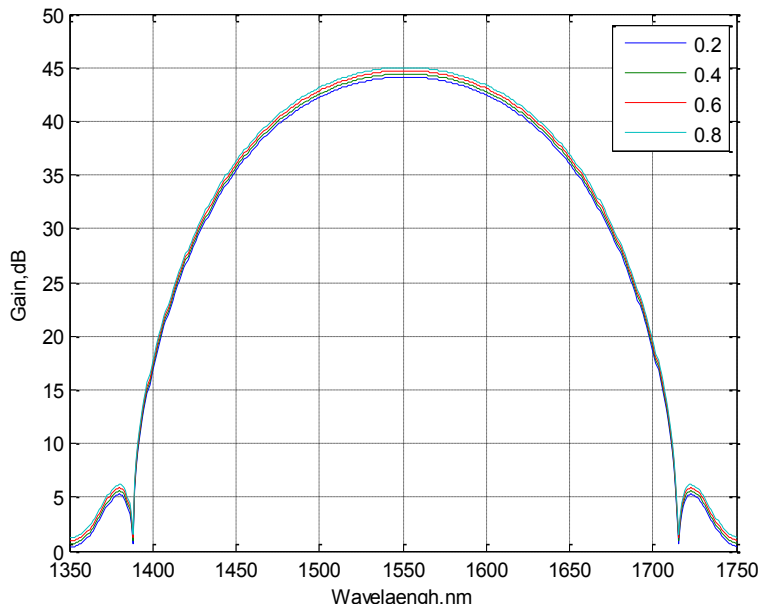

Figure 6: Variations of gain against wavelength at different values of attenuation $\alpha$

\section{Effect of dual pumping power on gain and bandwidth}

Figure 7 shows the relation between the amplifier gain and wavelength at different values of dual pumping power and the figure draw at assumed set of operating parameters attenuation constant $\alpha=0.1 \mathrm{~dB} / \mathrm{km}$, dual pump wavelengths $\lambda_{1}=1546 \mathrm{~nm}$ and $\lambda_{2}=1557 \mathrm{~nm}$, fiber length $\mathrm{L}=0.15 \mathrm{~km}$, non-linear coefficient $\gamma=25 \mathrm{w} / \mathrm{km}$ and phase mismatched $\beta=0.0006 \mathrm{~s}^{3} / \mathrm{km}$. In this case we adjustment the dual pumping power with the assumed set of operating parameters to obtain the maximum gain and bandwidth. The optimum results occurs at dual pumping power $\mathrm{p}_{1}=0.6 \mathrm{~mW}$ and $\mathrm{p}_{2}=0.6 \mathrm{~mW}$ where, the maximum gain is $60.5954 \mathrm{~dB}$ and the maximum bandwidth is $348 \mathrm{~nm}$.

\section{Optimum results of gain and bandwidth}

This Figure 8 shows the variations of the gain of the amplifier against the wavelength. After studying the effect of all parameters affecting on the gain and bandwidth this is conclusion of previous study of all parameters. In this case we adjustment a set of operating parameters as shown in Table 1 to obtained maximum gain and bandwidth.

We can get maximum gain $=61.6454 \mathrm{~dB}$ and bandwidth $\mathrm{BW}=350 \mathrm{~nm}$. 


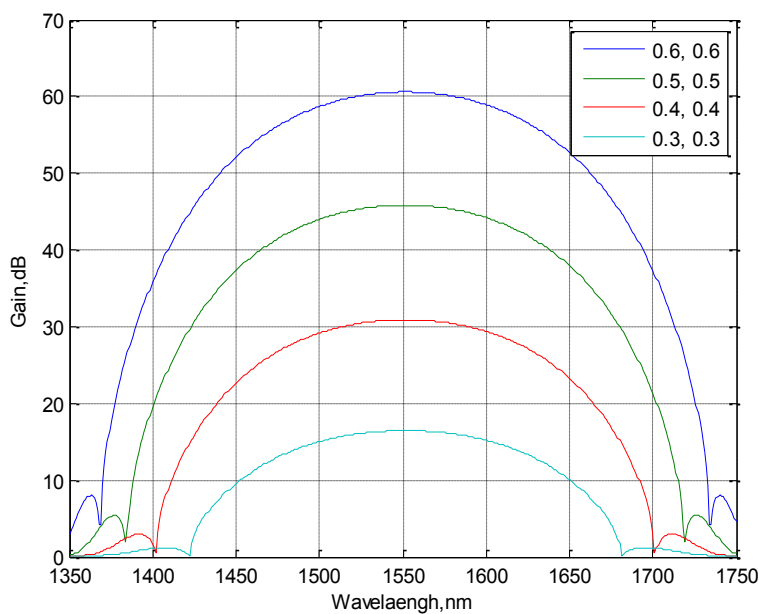

Figure 7: Variations of gain against wavelength at different values of dual pumping power.

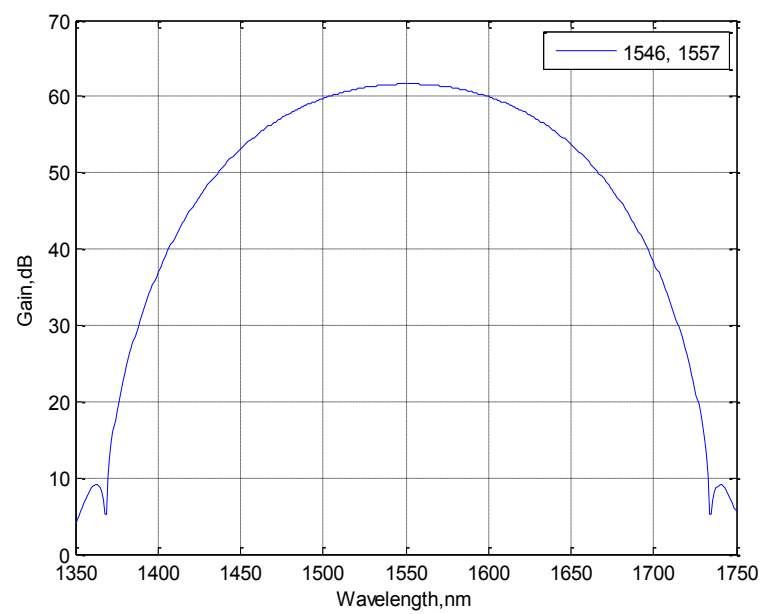

Figure 8: Variations of Gain against wavelength for assumed set of operating parameters at dual pump wavelengths $\lambda_{1}=1546 \mathrm{~nm}$ and $\lambda_{2}=1557 \mathrm{~nm}$.

\begin{tabular}{|c|c|c|c|}
\hline $\mathbf{1 1}$ & $\mathbf{1 5 4 6} \mathbf{~ n m}$ & $\mathbf{\lambda 2}$ & $\mathbf{1 5 5 7} \mathbf{~ n m}$ \\
\hline $\mathrm{P} 1$ & $0.6 \mathrm{~W}$ & $\mathrm{P} 2$ & $0.6 \mathrm{~W}$ \\
\hline $\mathrm{L}$ & $0.15 \mathrm{~km}$ & $\beta$ & $0.0006 \mathrm{~s} 3 / \mathrm{km}$ \\
\hline$\alpha$ & $0.8 \mathrm{~dB} / \mathrm{km}$ & $\mathrm{Y}$ & $25 \mathrm{w} / \mathrm{km}$ \\
\hline
\end{tabular}

Table 1: A set of operating parameters that uses in simulation.

\section{Conclusions}

In this work, we have investigated dual pump parametric amplifiers for gain variation using analytical model. The analysis shows feasibility of dual pump parametric amplifiers as wideband amplifiers with large gain. It was found that the center of FOPA with respect to zero wavelength is very important property to enhance the FOPA gain. By properly selecting the pump wavelengths and associated powers we showed that we can tailor the amplifier to demonstrate $61.6454 \mathrm{~dB}$ gains, with a $350 \mathrm{~nm}$ bandwidth. We have shown the factors that affect the gain in FOPAs. It was also found out that the gain of a FOPA is dependent on fiber length, dual pump wavelength and pump power, phase mismatch, nonlinear coefficient and attenuation. Therefore, the magnitude and shape of the gain can be optimized by tuning the fiber of all parameter values. These results should help in improving the transmission capacity in WDM and parametric amplification in long haul systems in fiber optic communication.

\section{References}

1. Rotich Kipnoo EK, Waswa D, Amolo G, Leitch AWR (2014) Gain Analysis for a 2-Pump Fiber Optical Parametric Amplifier. The African Review of Physics, pp. 47-52.

2. Tuan TH, Samuel E, Cheng T, Asano K, Suzuki T, et al. (2015) Optical parametric amplification in dual-pumped telluride hybrid microstructure optical fiber with engineered chromatic dispersion. Journal of Physics: Conference Series 619: 1-4.

3. Myint S, Lwin ZM, Tun HM (2015) Performance Analysis of Single-Pumped and Dual-Pumped Parametric Optical Amplifier. International Journal of Scientific \& Technology Research 4: 381- 386.

4. Boggio JMC, Marconi JD, Fragnito HL (2005) Double-pumped fiber optical parametric amplifier with flat gain over 47-nm bandwidth using a conventional dispersion-shifted fiber. IEEE Photonics Tech Lett 17: 1842-1844.

5. Zhang L, Liu B, Xin X, Liu L (2014) Fiber Optical Parametric Amplified Optical Direct-Detection OFDM Signal with Intensity Modulation Transfer Blocking. Opt Express 22: 25580-25586.

6. Kaminow P, Li T (2002) Optical Fibre Telecommunications IV B Systems and Impairment. Academic Press, Fourth edition.

7. Kaur G, Kaur G, Sharma S (2016) Performance Investigation of Dual pump Fiber Optical Parametric amplifier for Flat gain over $220 \mathrm{~nm}$ Gain Bandwidth. Int J Eng Sci 17: 451-457.

8. Vedadi AMA, Jadidi MM, Salehi JA (2012) Theoretical study of high repetition rate short pulse generation with fiber optical parametric amplification. J Lightwave Technol 30: 1263-1268.

9. Amin Shoaie M, Mohajerin-Ariaei A, Vedadi A, Camille-Sophie Brès (2014) Wideband generation of pulses in dual-pump optical parametric amplifier: theory and experiment. Opt Express 22: 4606-4619.

10. Effenberger FJ (2009) Next Generation PON-Part II: Candidate Systems for Next-Generation PON. IEEE Commun Mag 47: 50-57.

11. Salman MH, Hassan AH, Yasser HA (2013) Theoretical Calibration of DualPumps Fiber Optical Parametric Amplifier. International Journal of Application or Innovation in Engineering \& Management (IJAIEM) 2: 113-119. 